\title{
COMPACTIFICATIONS WITH DISCRETE REMAINDERS
}

\author{
JAMES P. HATZENBUHLER AND DON A. MATTSON
}

(Communicated by Franklin D. Tall)

\begin{abstract}
Conditions are obtained which characterize when a space has a Hausdorff compactification with a discrete remainder. A characterization is also given for when the minimal perfect compactification of a 0 -space has a discrete remainder. It is shown that a metric space has a compactification with a discrete remainder if and only if it is rimcompact. In general, however, for a space to have a compactification with a discrete remainder, it is not necessary that the space be rimcompact.
\end{abstract}

\section{INTRODUCTION}

In this paper all topological spaces are completely regular and Hausdorff and all compactifications are Hausdorff. A remainder of a compactification $\alpha X$ of $X$ is the space $\alpha X-X$. A major problem in compactification theory is to determine when, for each $X$ in a certain class of spaces, there is a member of another class of spaces which can serve as a remainder of $X$. (See [1], [2], [6], [9], and [13], for example.) The aim of this paper is to characterize the class of spaces $X$ which admit compactifications $\alpha X$ such that $\alpha X-X$ is discrete. The problem is trivial for locally compact spaces, hence hereafter we consider only nonlocally compact spaces. Recall that the residue $R(X)$ of $X$ is the set of points in $X$ which do not possess compact neighborhoods. To characterize when $X$ has a discrete remainder, then, it is natural to seek conditions which involve $R(X)$. Since $\mathrm{Cl}_{\alpha X}(\alpha X-X)=(\alpha X-X) \cup R(X)$, in order that $\alpha X-X$ be discrete, it is necessary that $R(X)$ be compact.

A space $X$ is a 0 -space if $X$ has a compactification with 0-dimensional remainder. Any 0 -space has a compactification $\phi X$ which is the maximal compactification with 0 -dimensional remainder. A space $X$ is rimcompact if it has a base of open sets $O$ with compact boundaries $\operatorname{Fr}_{X} O$, or $\pi$-open sets. Every rimcompact $X$ is a 0 -space and in this case $\phi X$ is called the Freudenthal compactification of $X$. Clearly, for $X$ to admit a discrete remainder, it is also necessary that $X$ be a 0 -space.

Received by the editors February 17, 1994.

1991 Mathematics Subject Classification. Primary 54D40.

Key words and phrases. Discrete remainders, perfect compactifications, rimcompact, metric spaces.

(C) 1995 American Mathematical Society 
A compactification $\alpha X$ of $X$ is perfect if, for each open subset $O$ of $X$, $\mathrm{Cl}_{\alpha X}\left(\operatorname{Fr}_{X} O\right)=\operatorname{Fr}_{\alpha X} U$, where $U$ is the largest $\alpha X$-open set satisfying $U \cap X=$ $O$. Recall that $\phi X$ is the smallest perfect compactification of $X$ and that $\beta X$, the Stone-Čech compactification, is the largest perfect compactification. See [9], [10], and [12] for properties of perfect compactifications. Also, $\phi X$ is obtained from $\beta X$ by identifying the components of $\beta X-X$ to points. (See [10] and [12] for further discussion.)

Terada has shown in [13] that a metric space with compact residue has a countable discrete remainder if and only if it is rimcompact and Čech complete. In $\S 2$ of this paper we provide conditions, internal to $X$, which characterize when any $X$ admits a discrete remainder. Examples show that when a space has a discrete remainder it need not have a maximal compactification with this property. However, when $X$ has a maximal compactification with a discrete remainder, then this compactification is $\phi X$. Accordingly, we also characterize when $\phi X-X$ is discrete.

In $\S 4$ we show that for metric spaces (with compact residue) it is precisely the class of rimcompact spaces which have discrete remainders and all such remainders are countable.

Finally, we provide examples to illuminate the results and, in particular, to show that, in contrast to the case for metric spaces, a nonrimcompact space $X$ can have an uncountable discrete remainder so that $X$ is also not Ceech complete.

\section{THE MAIN THEOREM}

For $f \in C(X)$, where $C(X)$ is the ring of continuous real-valued functions on $X$, the zero-set of $f$ is denoted by $Z(f)$. A nonempty family $\mathscr{F}$ of nonempty zero-sets of a space $X$ which is closed under pairwise intersection is a $z$-filter provided any zero-set is in $\mathscr{F}$ whenever it contains an element of $\mathscr{F}$. A $z$-ultrafilter is a maximal $z$-filter. Zero-sets and related concepts are studied extensively in [7]. By $C^{*}(X)$ we indicate the subring of bounded functions in $C(X)$. For any $\alpha X$ and open set $O$ in $X$, the extension of $O$ (to $\alpha X$ ) is $\operatorname{Ex}_{\alpha} O=\alpha X-\mathrm{Cl}_{\alpha}(X-O)$ and is the largest open set in $\alpha X$ whose trace on $X$ is $O$. Moreover, if $\alpha X$ is perfect and $O$ is $\pi$-open, then $\mathrm{Cl}_{\alpha} O=\mathrm{Cl}_{X} O \cup \mathrm{Ex}_{\alpha} O$. See [3] for other properties of extensions.

Lemma 2.1. Let $F$ be a closed, noncompact locally compact subset of $X$ with compact boundary and let $O$ be open in $X$. Then

(A) $F-O$ compact implies $\mathrm{Cl}_{\beta} F-F \subseteq \operatorname{Ex}_{\beta} O$.

(B) $F \cap \mathrm{Cl}_{X} O$ compact implies $\mathrm{Cl}_{\beta} F-F \subseteq \mathrm{Ex}_{\beta}\left(X-\mathrm{Cl}_{X} O\right)$.

Proof. (A) If $F-O$ is compact, then all points of $\mathrm{Cl}_{\beta} F-F$ are limit points of $F \cap O$. Since $\beta X$ is perfect and $\operatorname{Fr}_{X} F$ is compact, $U=\mathrm{Cl}_{\beta} F-$ $\left[\operatorname{Fr}_{X} F \cup(F-O)\right]$ is open in $\beta X$ and $U \cap X \subseteq O$. Now $U \cup \operatorname{Ex}_{\beta} O$ is a $\beta X$-open set whose trace on $X$ is $O$. Since $\operatorname{Ex}_{\beta} O$ is maximal with respect to this property, it follows that $\mathrm{Cl}_{\beta} F-F \subseteq \operatorname{Ex}_{\beta} O$.

(B) follows similarly and the proof is complete.

The main result of this section affords a characterization, internal to $X$, of when any $\alpha X$ has discrete remainders. We denote the space of positive integers by $N$ and the closed unit interval by $I$, each equipped with its usual topology. 
Theorem 2.2. Suppose a space $X$ has compact residue $R(X)$. Then the following are equivalent:

(A) $X$ has a compactification with a discrete remainder.

(B) $X$ contains a family $\left\{Z_{\alpha} \mid \alpha \in A\right\}$ of locally compact, noncompact zerosets which satisfy

(i) $\operatorname{Fr}_{X} Z_{\alpha}$ is compact;

(ii) $Z_{\alpha} \cap Z_{\gamma}$ is compact for $\alpha \neq \gamma$;

(iii) every free $z$-ultrafilter in $X$ contains some $Z_{\alpha}$;

(iv) for each $x \neq y$ in $R(X)$, there exist open $O_{x y}$ and $U_{x y}$ such that $x \in O_{x y}, y \notin \mathrm{Cl}_{X} O_{x y}, x$ and $y \notin \mathrm{Cl}_{X} U_{x y}$, and for all $Z_{\alpha}, Z_{\alpha}-O_{x y}, Z_{\alpha} \cap$ $\mathrm{Cl}_{X} O_{x y}$ or $Z_{\alpha}-U_{x y}$ is compact.

Proof. (A) implies (B). Suppose $\delta X-X$ is discrete. For each point $p \in \delta X-X$, there is a $\delta X$-neighborhood $N_{p}$ of $p$ such that $N_{p} \cap(\delta X-X)=\{p\}$. Choose a continuous mapping $f_{p}$ of $\delta X$ into $I$ such that $f_{p}(p)=0$ and $f_{p}\left(\delta X-N_{p}\right)=$ 1. Let $Z_{p}=f_{p}^{-1}[0,1 / 4] \cap X$, a zero-set in $X$. Then $\operatorname{Fr}_{X} Z_{p} \subseteq f_{p}^{-1}(1 / 4) \subseteq X$, hence $\operatorname{Fr}_{X} Z_{p}$ is compact. Now $\left\{Z_{p} \mid p \in \delta X-X\right\}$ is a collection of noncompact, locally compact zero-sets which satisfies $(B)(\mathrm{i})$.

For $p \neq q$ in $\delta X-X$ we have

$$
f_{p}^{-1}[0,1 / 4] \cap f_{q}^{-1}[0,1 / 4]=Z_{p} \cap Z_{q} \subseteq X,
$$

so $Z_{p} \cap Z_{q}$ is compact and (B)(ii) holds.

Next, let $\mathscr{F}$ be any free $z$-ultrafilter in $X$. Then $\mathscr{F}$ converges to a point $y \in \beta X-X$. If $t$ is the canonical mapping of $\beta X$ onto $\delta X$, then $t(y)=$ $q \in \delta X-X$ and $t^{-1}\left(f_{q}^{-1}[0,1 / 4]\right)$ is a $\beta X$-neighborhood of $y$. Hence $Z_{q}=$ $t^{-1}\left(f_{q}^{-1}[0,1 / 4]\right) \cap X$ contains a member $Z$ of $\mathscr{F}$. This implies $Z_{q} \in \mathscr{F}$ and (B)(iii) holds.

For $x \neq y$ in $R(X)$, select $\widehat{O}_{x y}$, open in $\delta X$, such that $x \in \widehat{O}_{x y}$ and $y \notin \mathrm{Cl}_{\delta} \widehat{O}_{x y}$. Let $A=\mathrm{Cl}_{\delta}\left\{y \in \delta X-X \mid y \in \mathrm{Fr}_{\delta X} \widehat{O}_{x y}\right\}$, a compact set with $x, y \notin A$. Choose any $\delta X$-open $\widehat{U}_{x y}$ for which $A \subseteq \widehat{U}_{x y}$ and $x, y \notin \mathrm{Cl}_{\delta} \widehat{U}_{x y}$. Set $O_{x y}=\widehat{O}_{x y} \cap X$ and $U_{x y}=\widehat{U}_{x y} \cap X$. Now suppose some $Z_{p}$ satisfies $Z_{p}-O_{x y}$ and $Z_{p} \cap \mathrm{Cl}_{X} O_{x y}$ are noncompact. Then $p \notin \operatorname{Ex}_{\delta} O_{x y}$ and $p \notin$ $\operatorname{Ex}_{\delta}\left(X-\mathrm{Cl}_{X} O_{x y}\right)$, hence $p \in A$. Thus $p \in \operatorname{Ex}_{\delta} U_{x y}$ so that $Z_{p}-U_{x y}$ is compact and (B)(iv) holds.

(B) implies (A). For each $\alpha \in A$, let $F_{\alpha}=\mathrm{Cl}_{\beta} Z_{\alpha}-Z_{\alpha}$. Then each $F_{\alpha}$ is compact, and since $\beta X$ is perfect, each $F_{\alpha}$ is open in $\beta X-X$. Suppose $y \in F_{\alpha} \cap F_{\gamma}$, for $\alpha \neq \gamma$. Then $Z_{\alpha}$ has compact boundary and int $Z_{\alpha} \neq \phi$, so $y \in \operatorname{Ex}_{\beta}\left(\right.$ int $\left._{X} Z_{\alpha}\right)$, a $\beta X$-open set. Now $N_{y}=\operatorname{Ex}_{\beta}\left(\right.$ int $\left._{X} Z_{\alpha}\right)-\left(Z_{\alpha} \cap Z_{\gamma}\right)$ is a $\beta X$-neighborhood of $y$ which misses $Z_{\gamma}$, a contradiction. Hence $F_{\alpha} \cap F_{\gamma}=\phi$ whenever $\alpha \neq \gamma$.

Take any point $y \in \beta X-X$. Since a free $z$-ultrafilter $\mathscr{F}$ in $X$ converges to $y$ and by (B)(iii) some $Z_{\alpha} \in \mathscr{F}$, we have $y \in F_{\alpha}$. Thus the family $\left\{F_{\alpha} \mid \alpha \in A\right\}$ is a partition of $\beta X-X$ into compact $(\beta X-X)$-open sets.

Next, for $x \neq y$ in $R(X)$ choose $O_{x y}$ and $U_{x y}$ as in (B)(iv). We show that there exist disjoint neighborhoods of $x$ and $y$, respectively, which "split" along the $F_{\alpha}$ 's. If $Z_{\alpha}-O_{x y}$ is compact, Lemma 2.1 provides that $F_{\alpha} \subseteq \operatorname{Ex}_{\beta} O_{x y}$. A similar condition holds when $Z_{\alpha} \cap \mathrm{Cl}_{X} O_{x y}$ is compact. Observe that $\operatorname{Ex}_{\beta} O_{x y}$ and $\operatorname{Ex}_{\beta}\left(X-\mathrm{Cl}_{X} O_{x y}\right)$ are disjoint, and suppose that some $F_{\alpha}$ meets both sets. Then both $Z_{\alpha}-O_{x y}$ and $Z_{\alpha} \cap \mathrm{Cl}_{X} O_{x y}$ are noncompact, hence $Z_{\alpha}-U_{x y}$ is 
compact. Thus, $F_{\alpha} \subseteq \operatorname{Ex}_{\beta} U_{x y}$ by Lemma 2.1. Let

$$
B=\mathrm{Cl}_{\beta}\left[\bigcup\left\{F_{\alpha} \mid F_{\alpha} \subseteq \operatorname{Ex}_{\beta} U_{x y}\right\}\right] .
$$

Then $B \subseteq \mathrm{Cl}_{\beta} \operatorname{Ex}_{\beta} U_{x y}$ and $x, y \notin B$. Now $\widehat{O}_{x}=\operatorname{Ex}_{\beta} O_{x y}-B$ and $\widehat{O}_{y}=$ $\operatorname{Ex}_{\beta}\left(X-\mathrm{Cl}_{X} O_{x y}\right)-B$ are $\beta X$-open disjoint sets containing $x$ and $y$, respectively. Moreover, $O_{x}$ satisfies the condition that $F_{\alpha} \cap \widehat{O}_{x} \neq \phi$ implies $F_{\alpha} \subseteq \widehat{O}_{x}$, and $O_{y}$ satisfies a similar condition.

Finally, if $t$ is the projection of $\beta X$ onto the quotient space $\beta X / C$ taken with respect to the decomposition $C=\left\{F_{\alpha} \mid \alpha \in A\right\} \cup\{\{x\} \mid x \in X\}$, then $t$ satisfies $t^{-1}\left(t\left(\widehat{O}_{x}\right)\right)=\widehat{O}_{x}$ and $t^{-1}\left(t\left(\widehat{O}_{y}\right)\right)=\widehat{O}_{y}$. This is enough to ensure that $\beta X / C$ is a Hausdorff compactification of $X$ with discrete remainder. This completes the proof.

We note in [8] that condition (B)(iii) of 2.2 can be replaced by the following equivalent condition: for each $X$-closed subset $F$ of $X-R(X)$ there exists finitely many $Z_{\alpha_{i}}$ such that $F-\bigcup\left\{\right.$ int $\left._{X} Z_{\alpha_{i}} \mid i=1, \ldots, n\right\}$ is compact.

\section{TWo SPECIAL CASES}

Here we characterize when $\phi X-X$ is discrete, which occurs if and only if $X$ has a maximal compactification with a discrete remainder. Also, we consider the case when $R(X)$ is totally disconnected and compact. 0-spaces having this property are rimcompact by 2.4 of [4] so that $\phi X$ is the Freudenthal compactification. In both cases we obtain characterizations having sharpened versions of (B)(iv) of 2.2. We first consider an example. Let $W$ be the space of all countable ordinals equipped with the usual topology and $W^{*}=\beta W$, so that $W^{*}-W=\left\{\omega_{1}\right\}$, where $\omega_{1}$ is the first uncountable ordinal. Take $Y=\{1 / n \mid n \in N\} \cup\{0\}$ and set $X=W \times Y$. Then $\beta X=\phi X=W^{*} \times Y$ and it is clear that $X$ has discrete remainders but no maximal compactification with discrete remainder.

Theorem 3.1. Suppose $X$ is 0-space with $R(X)$ compact. Then $\phi X-X$ is discrete if and only if there is a family $\left\{Z_{\alpha} \mid \alpha \in A\right\}$ of locally compact, noncompact zero-sets satisfying

(i) $\operatorname{Fr}_{X} Z_{\alpha}$ is compact;

(ii) $Z_{\alpha} \cap Z_{\gamma}$ is compact when $\alpha \neq \gamma$;

(iii) every free $z$-ultrafilter in $X$ contains some $Z_{\alpha}$;

(iv) for each $\pi$-open set $O$ in $X$, either $Z_{\alpha}-O$ or $Z_{\alpha} \cap \mathrm{Cl}_{X} O$ is compact, for all $\alpha \in A$.

Proof. Obtain a family $\left\{Z_{p} \mid p \in \phi X-X\right\}$ of zero-sets which satisfies (i)-(iii) as in the proof of 2.2. For (iv), let $O$ be any $\pi$-open set in $X$. Since $\phi X$ is perfect, the sets $\operatorname{Ex}_{\phi} O \cap(\phi X-X)$ and $\operatorname{Ex}_{\phi}\left(X-\mathrm{Cl}_{X} O\right) \cap(\phi X-X)$ partition $\phi X-X$; hence for $p \in \phi X-X$, either $p \in \operatorname{Ex}_{\phi} O$ or $p \in \operatorname{Ex}_{\phi}\left(X-\mathrm{Cl}_{X} O\right)$. If $p \in \operatorname{Ex}_{\phi} O$, since $p$ is the only limit point of $Z_{p}$ in $\phi X-X$, we have $Z_{p}-\operatorname{Ex}_{\phi} O=Z_{p}-O$ compact. Similarly, $p \in \operatorname{Ex}_{\phi}\left(X-\mathrm{Cl}_{X} O\right)$ implies $Z_{p} \cap \mathrm{Cl}_{X} O$ is compact, so that (iv) holds.

Now assume $\left\{Z_{\alpha} \mid \alpha \in A\right\}$ is a family of noncompact, locally compact zerosets in $X$ satisfying (i)-(iv). As in the proof of 2.2 , take $F_{\alpha}=\mathrm{Cl}_{\beta} Z_{\alpha}-Z_{\alpha}$, so that $\left\{F_{\alpha} \mid \alpha \in A\right\}$ is a partition of $\beta X-X$ into compact sets which are open in $\beta X-X$. We show that each $F_{\alpha}$ is a component of $\beta X-X$. 
Let $t$ be the canonical mapping of $\beta X$ into $\phi X$. Suppose $F_{\alpha}=A \cup B$, where $A$ and $B$ are disjoint, nonempty, and open in $\beta X-X$. Since $t$ identifies components of $\beta X-X$ to points, for $a \in A$ and $b \in B$ we have $t(a) \neq t(b)$ in $\phi X-X$. Also, $R(X)$ is compact, so $X$ is almost rimcompact (see Theorem 2.8 of [3]), hence there is a $\pi$-open set $O$ in $X$ for which $t(a) \in \operatorname{Ex}_{\phi} O$ and $t(b) \in \operatorname{Ex}_{\phi}\left(X-\mathrm{Cl}_{X} O\right)$. But $a \in t^{-1}\left(\operatorname{Ex}_{\phi} O\right)$ implies $\mathrm{Cl}_{X} O \cap Z_{\alpha}$ is not compact and $b \in t^{-1}\left(\operatorname{Ex}_{\phi}\left(X-\mathrm{Cl}_{X} O\right)\right)$ implies $Z_{\alpha}-O$ is not compact, which contradicts (iv). Hence $F_{\alpha}$ is connected, and since $F_{\alpha}$ is compact and $(\beta X-X)$-open, it is a component of $\beta X-X$, whence it follows that $\phi X-X$ is discrete. This completes the proof.

It is easy to find examples of spaces with $R(X)$ totally disconnected and compact which have discrete remainders, but for which $\phi X-X$ is not discrete. Such spaces possess families of zero-sets which satisfy (i)-(iv) of the following theorem but fail to satisfy (iv) of 3.1 .

Theorem 3.2. Assume $X$ has compact totally disconnected residue $R(X)$. Then $X$ has a compactification $\delta X$ with discrete remainder if and only if $X$ contains a family $\left\{Z_{\alpha} \mid \alpha \in A\right\}$ of noncompact, locally compact zero-sets satisfying (i)-(iii) of 3.1 and

(iv) there is a family of $\pi$-open sets $O$ which separate points of $R(X)$, and exactly one of $Z_{\alpha}-O$ or $Z_{\alpha} \cap \mathrm{Cl}_{X} O$ is compact, for all $\alpha \in A$.

Proof. Assume $\delta X-X$ is discrete and, as in 2.2, obtain a family of noncompact, locally compact zero-sets $\left\{Z_{r} \mid r \in \delta X-X\right\}$ which satisfy (i)-(iii) of 2.2. For (iv), let $p, q \in R(X)$. Let $Y=(\delta X-X) \cup R(X)$. Then $Y$ is compact and 0-dimensional. Thus there is a compact $Y$-open neighborhood $N_{p q}$ with $p \in N_{p q}$ and $q \notin N_{p q}$ and a continuous map $f_{p q}$ of $\delta X$ into $I$ satisfying $f_{p q}\left(N_{p q}\right)=0$ and $f_{p q}\left(Y-N_{p q}\right)=1$. Let $O_{p q}=f_{p q}^{-1}[0,1 / 2) \cap X$. It follows that $O_{p q}$ is $\pi$-open with $p \in O_{p q}$ and $q \notin \mathrm{Cl}_{X} O_{p q}$. Take any $Z_{r}$ in $\left\{Z_{r} \mid r \in \delta X-X\right\}$. Either $r \in N_{p q}$ or $r \in Y-N_{p q}$. When $r \in N_{p q}, f_{p q}(r)=0$. But $f_{p q} \geq 1 / 2$ on $Z_{r}-O_{p q}$, so that $r$ cannot be a limit point of $Z_{r}-O_{p q}$. Thus $Z_{r}-O_{p q}$ is compact, but $Z_{r} \cap \mathrm{Cl}_{X} O_{p q}$ is not. Similarly, when $r \in Y-N_{p q}$, $Z_{r} \cap \mathrm{Cl}_{X} O_{p q}$ is compact but $Z_{r}-O_{p q}$ is not. Hence, (iv) holds.

Now assume $\left\{Z_{\alpha} \mid \alpha \in A\right\}$ is a family of locally compact, noncompact zero sets satisfying (i)-(iv). As in 2.2 the sets $\left\{F_{\alpha} \mid \alpha \in A\right\}$, where $F_{\alpha}=\mathrm{Cl}_{\beta} Z_{\alpha}-Z_{\alpha}$, are a partition of $\beta X-X$ into compact open sets. For $p \neq q$ in $R(X)$, choose $\pi$-open $O$ according to (iv) which separates $p$ and $q$. If some $F_{\alpha}$ meets both $\operatorname{Ex}_{\beta} O$ and $\operatorname{Ex}_{\beta}\left(X-\mathrm{Cl}_{X} O\right)$, then both $Z_{\alpha} \cap \mathrm{Cl}_{X} O$ and $Z_{\alpha}-O$ are noncompact, which contradicts (iv). Thus, for any such $O$, either $F_{\alpha} \subseteq \operatorname{Ex}_{\beta} O$ or $F_{\alpha} \subseteq \operatorname{Ex}_{\beta}\left(X-\mathrm{Cl}_{X} O\right)$.

Now the decomposition $\left\{F_{\alpha} \mid \alpha \in A\right\} \cup\{\{x\} \mid x \in X\}$ of $\beta X$ into compact sets can be projected onto its associated quotient space to obtain a Hausdorff compactification $\delta X$ of $X$ for which $\delta X-X$ is discrete, and the proof is complete.

\section{METRIC SPACES WITH DISCRETE REMAINDERS}

Recall that $X$ is Čech complete whenever $X$ is a $G_{\delta}$-set in $\beta X$. In [13] Terada showed that a metric space $X$ with nonempty compact residue has a compactification with a countable discrete remainder if and only if $X$ is Čech 
complete and rimcompact. Here we show that a metric space $X$ with compact residue has a discrete remainder if and only if $X$ is rimcompact, or equivalently, $X$ is a 0 -space. Moreover, any discrete remainder of a metric space must be countable.

In general, a set $S$ in any space $X$ is said to have countable character whenever $S$ has a countable neighborhood base, and $X$ is Lindelöf at infinity if every compact set is contained in a compact set of countable character. (See [9, p. 113].)

We shall need the following result.

Proposition 4.1. Let $X$ be any space with compact $R(X)$. Then the following are equivalent:

(A) $X$ is Lindelöf at infinity;

(B) $\alpha X-X$ is a Lindelöf space, for all compactifications $\alpha X-X$ of $X$;

(C) $\alpha X-X$ is a $\sigma$-compact, for all compactifications $\alpha X$ of $X$;

(D) $X$ is Čech complete;

(E) $R(X)$ is contained in a compact set of countable character.

Proof. That (A) and (B) are equivalent is Theorem 35, Chapter VI of [9], and (B) and (C) are equivalent when $\alpha X-X$ is locally compact by Theorem 7.2 of $\mathrm{XI},[5]$. That (C) and (D) are equivalent is obvious, as is (A) implies (E).

Now assume (E) and let $K$ be a compact set of countable character containing $R(X)$. Let $\left\{G_{n} \mid n \in N\right\}$ be a countable open neighborhood base for $K$ and take $F_{n}=X-G_{n}$, for all $n \in N$. Then $\widehat{F}_{n}=\mathrm{Cl}_{\beta X} F_{n}-F_{n}$ is a compact subset of $\beta X-X$. For $p \in \beta X-X$, there is a free $z$-ultrafilter $\mathscr{F}$ in $X$ which converges to $p$. Since $K$ is compact, we can find $Z \in \mathscr{F}$ such that $Z \cap K=\phi$. Thus, $Z \subseteq X-G_{n}=F_{n}$, for some $n$, so that $p \in \mathrm{Cl}_{\beta} Z \subseteq \mathrm{Cl}_{\beta} F_{n}$. Hence $p \in \widehat{F}_{n}$, and $\left\{\widehat{F}_{n} \mid n \in N\right\}$ is a cover of $\beta X-X$ by compact sets which insures that $X$ is Čech complete. This completes the proof.

If $\delta X$ and $\gamma X$ are compactifications of any nonlocally compact $X$ with discrete remainders, then

$$
\operatorname{card}(\delta X-X)=\operatorname{card}(\gamma X-X) .
$$

To see this, let $t_{\delta}$ and $t_{\gamma}$ be the respective canonical mappings of $\beta X$ onto $\delta X$ and $\gamma X$. For each $p \in \delta X-X, t_{\delta}^{-1}(p)$ is a compact open subset of $\beta X-X$. Similarly, $t_{\gamma}^{-1}(q)$ is compact and open in $\beta X-X$ for each $q \in \gamma X-X$. Since at most finitely many $t_{\delta}^{-1}(p)$ 's can meet any $t_{\gamma}^{-1}(q)$ and vice versa, the result follows.

Note that in any metric space $X$ all compact sets have countable character, so if $X$ has compact residue, then $X$ is Čech complete. Thus, the requirement that $X$ be Čech complete can be omitted from Terada's theorem.

Using Terada's result and the fact that for metric spaces $X$ is a 0 -space if and only if $X$ is rimcompact, the following is now immediate.

Theorem 4.2. Let $X$ be a metric space with $R(X)$ compact. Then the following are equivalent:

(A) $X$ is a 0 -space;

(B) $X$ is rimcompact;

(C) $X$ has a discrete remainder of countable cardinality. 


\section{EXAMPLes}

The following example shows that for $X$ to have a discrete remainder it is not enough that $\phi X-X$ be 0 -dimensional and locally compact.

Example 5.1. According to 2.1 of [14] we can find a locally compact, zerodimensional $Y$ for which $\beta Y-Y=I$. Then for any infinite discrete space $Z$, there is no continuous mapping $t$ of $Y$ onto $Z$. For, if such $t$ exists, then $t$ has a continuous extension $t^{\beta}$ from $\beta Y$ onto $\beta Z$. It is readily seen that $t^{\beta}$ must carry $I$ onto $\beta Z-Z$, which is impossible since $Z$ is strongly 0 -dimensional and $\beta Z-Z$ is nontrivial.

Now set $X=W^{*} \times \beta Y-\left(\left\{\omega_{1}\right\} \times Y\right)$. Then $\beta X-X$ is 0 -dimensional and locally compact but there can be no mapping of $\beta X-X$ onto a discrete $\alpha X-X$.

In Example 5.2 we use Theorem 3.2 to show that $X$ does not possess a compactification with a discrete remainder.

Example 5.2. Let $Y=I \times I-\{(1 / 2,1 / n+1) \mid n \in N\}, T=W^{*} \times(I \times I)$ and $X=W^{*} \times Y$. For each ordinal $\alpha \leq \omega_{1}$, set $X_{\alpha}=\{\alpha\} \times Y$, so that each $X_{\alpha}$ is a copy of $Y$. For notational convenience let $p_{n}^{\alpha}=(\alpha,(1 / 2,1 / n+1))$, for all $\alpha \leq \omega_{1}$ and $n \in N$, and $p_{0}^{\alpha}=(\alpha,(1 / 2,0))$, for all $\alpha$. Clearly, $X$ is a 0 -space and since $R(X)=\left\{p_{0}^{\alpha} \mid \alpha \leq \omega_{1}\right\}$ is compact and totally disconnected, $X$ is rimcompact by 2.4 of [4].

Suppose some compactification $\delta X$ has discrete $\delta X-X$. Now there must exist a family of noncompact, locally compact zero-sets $\left\{Z_{\gamma} \mid \gamma \in A\right\}$ which satisfy conditions (i)-(iii) of Theorem 3.2. By (iii) of 3.2 each point $p_{n}^{\omega_{1}}$ must be contained in some $\mathrm{Cl}_{T} Z_{\gamma}$ which we denote by $Z_{\gamma(n)}$. Then $Z_{\gamma(n)}$ is the zero-set of some $f_{n} \in C^{*}(X)$. The values of $f_{n}$ on any $X_{\alpha}$ are determined uniquely by values of $f_{n}$ on a countable dense subset of $X_{\alpha}$ and since $f_{n}$ is "eventually constant" on a copy of $W^{*}$, one can find $\alpha(n)<\omega_{1}$ such that $f_{n}(\gamma,(x, y))=f_{n}(\mu,(x, y))$, for all $\gamma, \mu \geq \alpha(n)$. Let $\alpha_{0}=\sup _{n \in N} \alpha(n)<$ $\omega_{1}$.

Since $R(X)$ is compact and totally disconnected, condition (iv) of Theorem 3.2 applies. Accordingly, let $O$ be any $\pi$-open set which satisfies $p_{0}^{\omega_{1}} \in O$ and let $\widehat{O}$, open in $T$, satisfy $\widehat{O} \cap X=O$. We show that $O$ cannot separate $p_{0}^{\alpha_{0}}$ and $p_{0}^{\omega_{1}}$. Now there is a $k \in N$ such that $p_{n}^{\omega_{1}} \in \widehat{O}$, for all $n \geq k$. But if $O$ is a "separating" set according to (iv) of 3.2, we require $Z_{\gamma(n)}-O$ or $Z_{\gamma(n)} \cap \mathrm{Cl}_{X} O$ to be compact, for all $n \in N$. But $\mathrm{Cl}_{T} Z_{\gamma(n)}$ contains $p_{n}^{\alpha}$, for all $\alpha \geq \alpha_{0}$ and $\hat{O}$ contains $p_{n}^{\omega_{1}}$, hence $\mathrm{Cl}_{T} O$ contains $p_{n}^{\alpha}$, for all $\alpha \geq \alpha_{0}$ and $n \geq k$. It follows that $p_{0}^{\alpha} \in \mathrm{Cl}_{X} O$ for all $\alpha \geq \alpha_{0}$, so that, in particular, $O$ cannot separate $p_{0}^{\omega_{1}}$ and $p_{0}^{\alpha_{0}}$. This contradicts (iv) of 3.2, hence no $\delta X-X$ can be discrete.

Recall that for metric spaces, if $\delta X-X$ is discrete, then $X$ must be rimcompact and Čech complete. However, the following is an example of a space with a discrete remainder which is neither rimcompact nor Čech complete.

Example 5.3. As in 2.1 of [14], choose a maximal family $\mathscr{R}$ of almost disjoint infinite subsets of $N$ such that the space $N \cup \mathscr{R}$ satisfies $\beta(N \cup \mathscr{R})-(N \cup \mathscr{R})=$ $I$. Then $X=W^{*} \times \beta(N \cup \mathscr{R})-\left(\left\{\omega_{1}\right\} \times \mathscr{R}\right)$, so that $R(X)$ is a copy of $I$ and $\beta X-X=\phi X-X$ is a homeomorph of $\mathscr{R}$, hence is discrete. For 
notational clarity, subspaces of $\left\{\omega_{1}\right\} \times \beta(N \cup \mathscr{R})$ will be regarded as subspaces of $\beta(N \cup \mathscr{R})$. We show that the points $1 / 3$ and $2 / 3$ in $R(X)$ cannot be separated by a $\pi$-open set in $X$.

For, suppose $V$ is $\pi$-open in $X$ with $1 / 3 \in V$ and $2 / 3 \notin \mathrm{Cl}_{X} V$. Since $\beta X$ is perfect, for $\gamma \in \mathscr{R}=\beta X-X$, either $\gamma \in \operatorname{Ex}_{\beta} V$ or $\gamma \in \operatorname{Ex}_{\beta} W$, where $W=X-\mathrm{Cl}_{X} V$. Since $\operatorname{Ex}_{\beta} V$ and $\operatorname{Ex}_{\beta} W$ are disjoint, $\gamma$ is not a boundary point of $\widehat{V}=\operatorname{Ex}_{\beta} V \cap(N \cup \mathscr{R})$ nor of $\widehat{W}=\operatorname{Ex}_{\beta} W \cap(N \cup \mathscr{R})$. Also, no point of $N$ can be a boundary point of $\widehat{V}$ or $\widehat{W}$. Thus $\widehat{V}$ and $\widehat{W}$ are clopen and disjoint in $N \cup \mathscr{R}$, so that $\mathrm{Cl}_{\beta(N \cup \mathscr{R})} \widehat{V} \cap \mathrm{Cl}_{\beta(N \cup \mathscr{R})} \widehat{W}=\phi$. But $\mathrm{Cl}_{\beta(N \cup \mathscr{R})} \widehat{V}=\mathrm{Cl}_{\beta(N \cup \mathscr{R})}\left(\operatorname{Ex}_{\beta X} V \cap \beta(N \cup \mathscr{R})\right)$ contains $1 / 3$ and, similarly, $2 / 3 \in \mathrm{Cl}_{\beta(N \cup \mathscr{R})} \widehat{W}$. Since $\mathrm{Cl}_{\beta(N \cup \mathscr{R})} \widehat{V}$ is clopen in $\beta(N \cup \mathscr{R})$, its intersection with $I$ now disconnects $I$, a contradiction.

Thus, no such $V$ can exist and $X$ is not rimcompact. By Proposition 4.1 and the fact that $\mathscr{R}$ cannot be countable, it follows that $X$ also fails to be Čech complete.

\section{REFERENCES}

1. G. L. Cain, Countable compactifications, General Topology and its Relations to Modern Analysis and Algebra VI (Proc. Sixth Prague Topological Symposium, 1986), Heldermann Verlag, Berlin, 1988.

2. R. E. Chandler, Hausdorff compactifications, Lecture Notes in Pure and Appl. Math., vol. 23, Marcel Dekker, New York and Basel, 1976.

3. B. Diamond, Some properties of almost rimcompact spaces, Pacific J. Math. 118 (1985), 63-77.

4. B. Diamond, J. Hatzenbuhler, and D. Mattson, On when a 0-space is rimcompact, Topology Proc. 13 (1988), 189-201.

5. J. Dugundji, Topology, Allyn and Bacon, Boston, 1966.

6. W. Fleissner, J. Kulesza, and R. Levy, Remainders of normal spaces, Topology Appl. 49 (1993), 167-174.

7. L. Gillman and M. Jerison, Rings of continuous functions, Van Nostrand, New York, 1960.

8. J. Hatzenbuhler and D. Mattson, Paracompact and metrizable remainders (submitted).

9. J. R. Isbell, Uniform spaces, Math. Surveys Monographs, vol. 12, Amer. Math. Soc., Providence, RI, 1962.

10. J. R. McCartney, Maximum zero-dimensional compactifications, Math. Proc. Cambridge Philos. Soc. 68 (1970), 653-661.

11. M. Rayburn, On Hausdorff compactifications, Pacific J. Math. 44 (1973), 707-714.

12. E. G. Sklyarenko, Some questions in the theory of bicompactifications, Trans. Amer. Math. Soc. 58 (1966), 216-244.

13. T. Terada, On countable discrete compactifications, Topology Appl. 7 (1977), 321-327.

14. J. Terasawa, Spaces $N \cup \mathscr{R}$ and their dimensions, Topology Appl. 11 (1980), 93-102. 56563

Department of Mathematics, Moorhead State University, Moorhead, Minnesota

E-mail address: hatzenbu@mhdma.moorhead.msus.edu

E-mail address: mattson@mhd1.moorhead.msus.edu 